\title{
$\pi$-SACS: $\underline{\mathrm{pH}}$ Induced $\underline{\text { Self}}-\underline{A}$ ssembled $\underline{\text { Cell }} \underline{\text { Sheets }}$ Without the Need for Modified Surfaces
}

\author{
Alireza Shahin-Shamsabadi 1, P. Ravi Selvaganapathy 1,2 \\ 1 School of Biomedical Engineering, McMaster University, Canada \\ 2 Department of Mechanical Engineering, McMaster University, Canada \\ Supporting Information
}




\section{Methods}

Optimization of pH and treatment timing for media A and B: Optimization of $\mathrm{pH}$ for delamination started from neutral $\mathrm{pH}$ of culture medium (7.4-7.5) and decreasing the $\mathrm{pH}$ in case of medium A and increasing it for medium B by 0.5 increments. In each case a maximum of 20 min was considered to see the desired effect. Media with $\mathrm{pH}$ of 7 and 6.5 didn't cause delamination even after 20 min incubation but at $\mathrm{pH}$ of 6 delamination was observed after 5 min on the edges and was completed by 10 min of treatment. Immediately after delamination sheets were transferred to basic medium with $\mathrm{pH}$ of 8 and it was able to maintain flatness of sheets even after 10 min of treatment and no further increase in $\mathrm{pH}$ was needed. Treatment with either media for longer than 20 min resulted in higher number of dead cells as compared to minimum timing requirements which was confirmed by live/dead staining (Data not shown). Effect of extreme changes of $\mathrm{pH}$ on sheets was studied by treating sheets with either pure acetic acid or $0.1 \mathrm{M}$ sodium hydroxide that resulted in immediate disintegration of sheets (Data not shown). 
Table S1. Sequence of the used primers for qRT-PCR (5'to 3')

\begin{tabular}{|c|l|l|}
\hline Target Gene & Forward & Reverse \\
\hline Myogen & GCA ATG CAC TGG AGT TCG & ACG ATG GAC GTA AGG GAG TG \\
\hline MyoD & GCC TGA GCA AAG TGA ATG AG & GCA GAC CTT CGA TGT AGC G \\
\hline$\beta$-Actin & $\begin{array}{l}\text { CAT GGA GTC CTG GCA TCC ACG } \\
\text { AAA CT }\end{array}$ & $\begin{array}{c}\text { ATC TCC TTC TGC ATC CTG TCG GCA } \\
\text { TA }\end{array}$ \\
\hline GAPDH & ATG TTT GTG ATG GGT GTG AA & ATG CCA AAG TTG TCA TGG AT \\
\hline
\end{tabular}




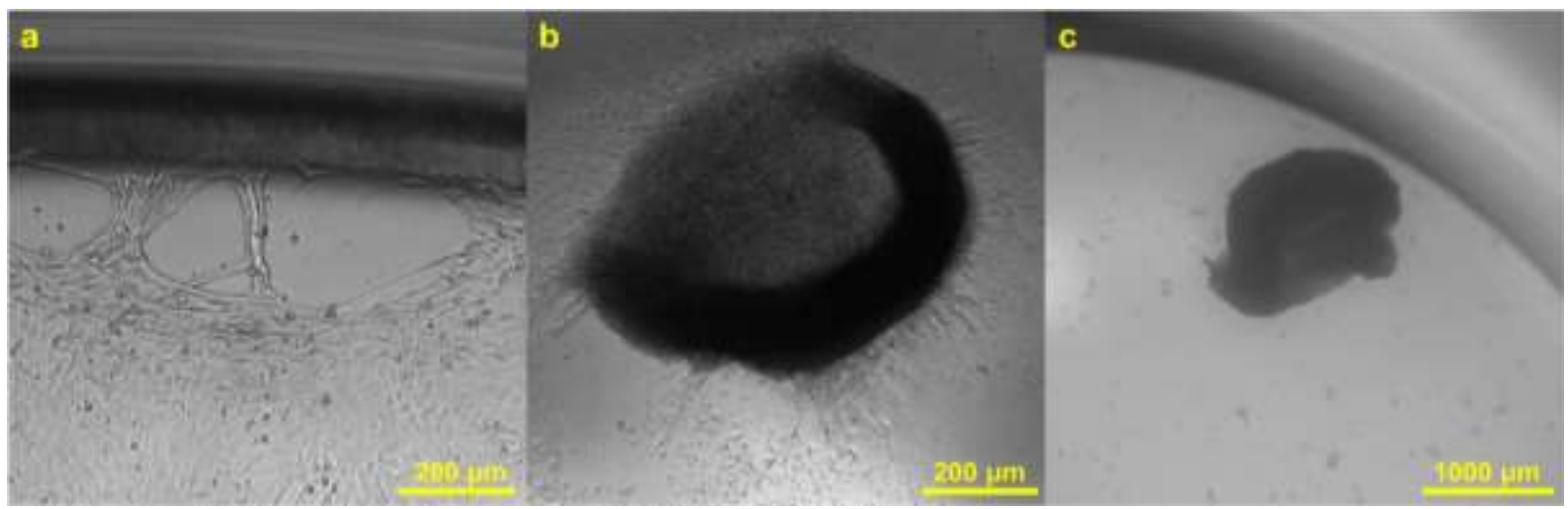

Figure S1. a) Cell traction force exerted at the edges of the wells; b) Advancement of traction to form undefined agglomerated structures that could lead to spheroid-like structures; c) Advancement of folding in case sheets are kept in medium A after delamination is completed.

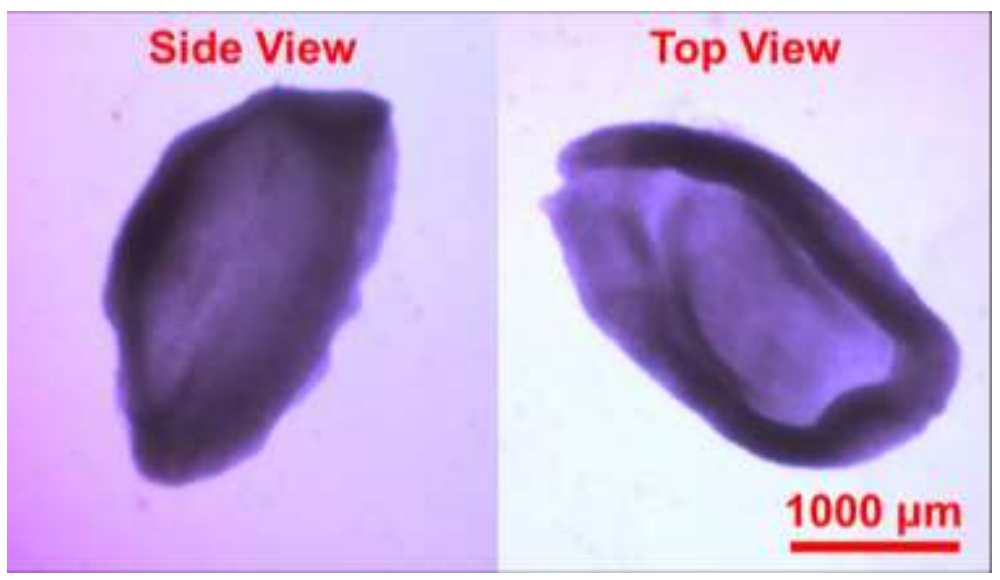

Figure S2. Deformed single-layer construct after 1 day from side and top views. Single-layer construct are more prone to unintended deformation 


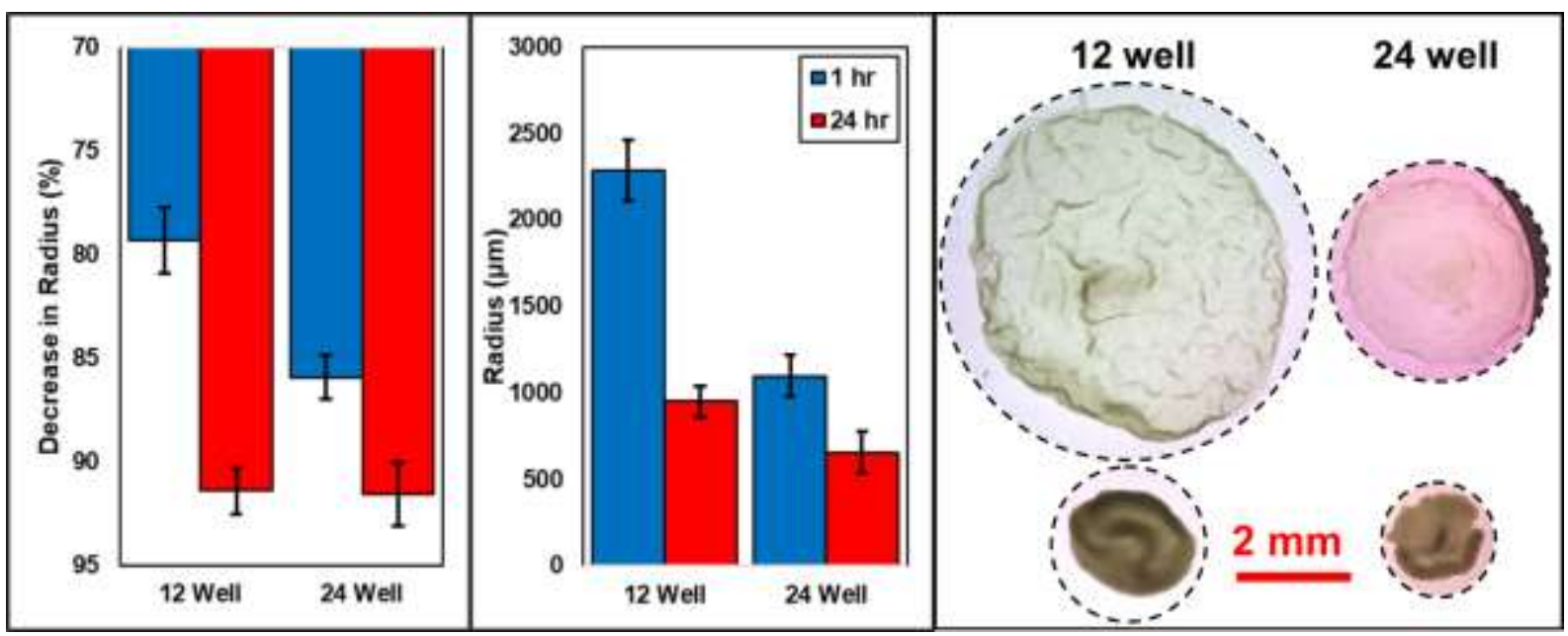

Figure S3. Difference between shrinkage of sheets formed with $0.235 \times 10^{6}$ cells in a 24 well plate and $0.480 \times 10^{6}$ cells in a 12 well plate. 


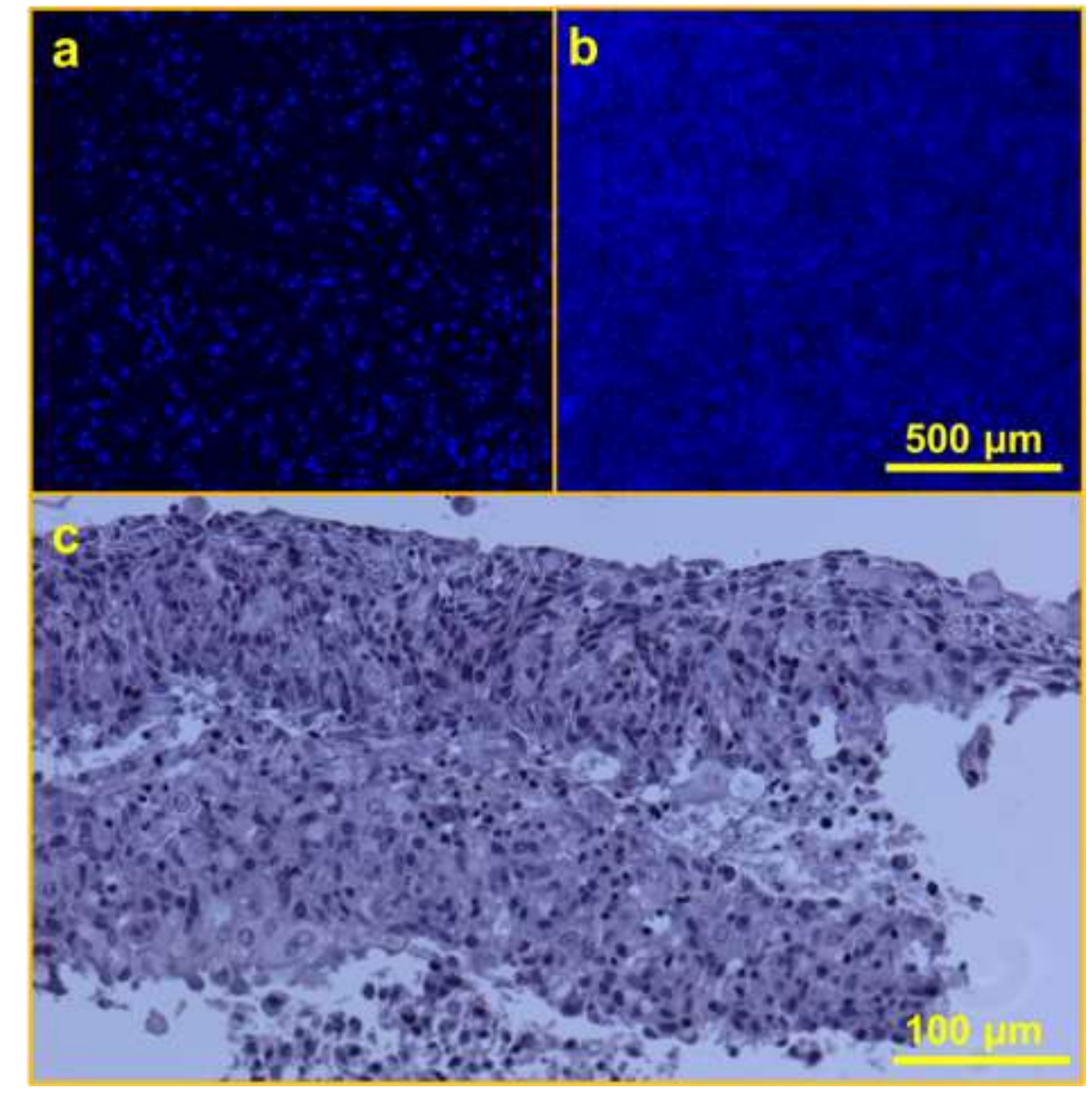

Figure S4. Difference between compactness of cells a) before and b) after delamination. In accordance with decrease in sheet size, cell compactness increases. c) H\&E stained section of a quadruple-layer construct. While in some locations layers are slightly separated from each other during the sectioning process, in most positions no clear interface was detectable between stacked layers. 


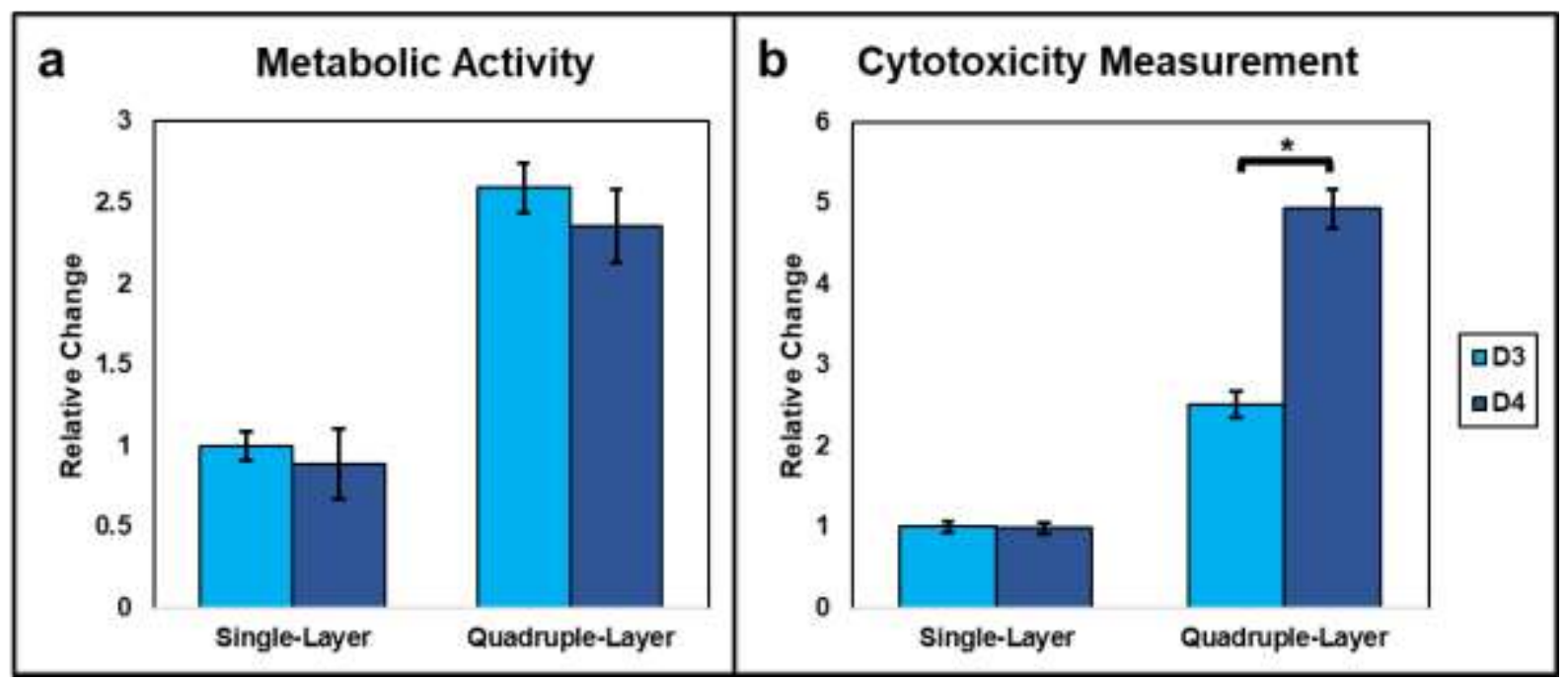

Figure S5. Long-term effect of delamination and stacking multiple layers on cell a) viability and b) cytotoxicity. Assays are performed one day after delamination (D3 in the whole process) and two days after (D4 of the process). P-value $<0.01$ for $n=4$. 


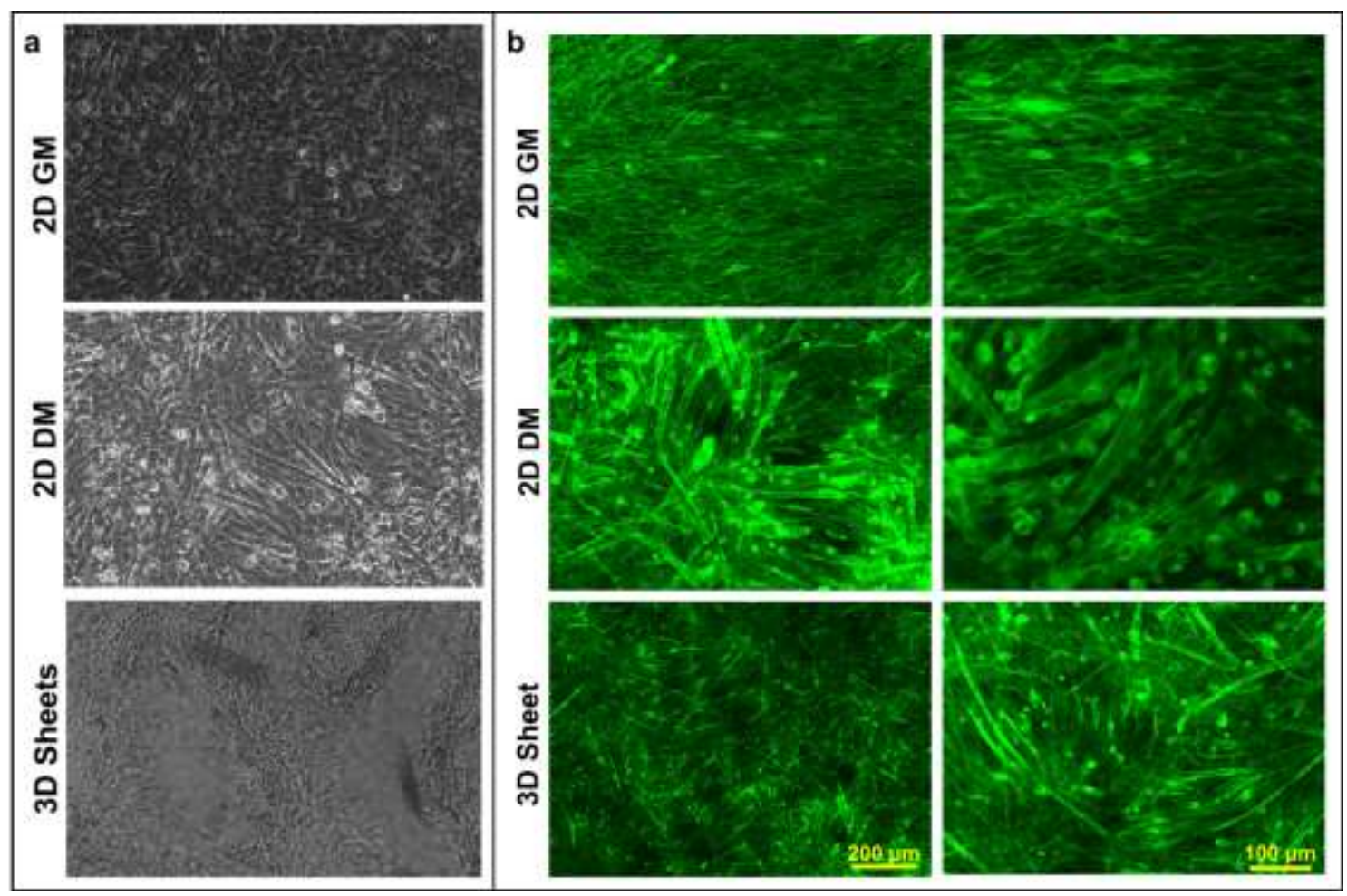

Figure S6. a) Phase contrast and b) fluorescent stained F-actin (using phalloidin) images of cells without differentiation (in GM), and with differentiation (in DM), and 3D sheets after delamination. Images are taken at D3 before delamination using $4 \mathrm{X}$ and/or 10X magnifications. Cells in differentiated state are elongated and has formed thick myofibers. For staining with phalloidin samples were fixed with $2 \%$ paraformaldehyde and after washing with PBS, staining was performed using 1:40 dilution of phalloidin (Alexa Fluor ${ }^{\mathrm{TM}} 488$ Phalloidin, Thermofisher, A12379) stock solution (300 units in $1 \mathrm{~mL}$ methanol) in PBS with $1 \mathrm{hr}$ incubation at room temperature. 


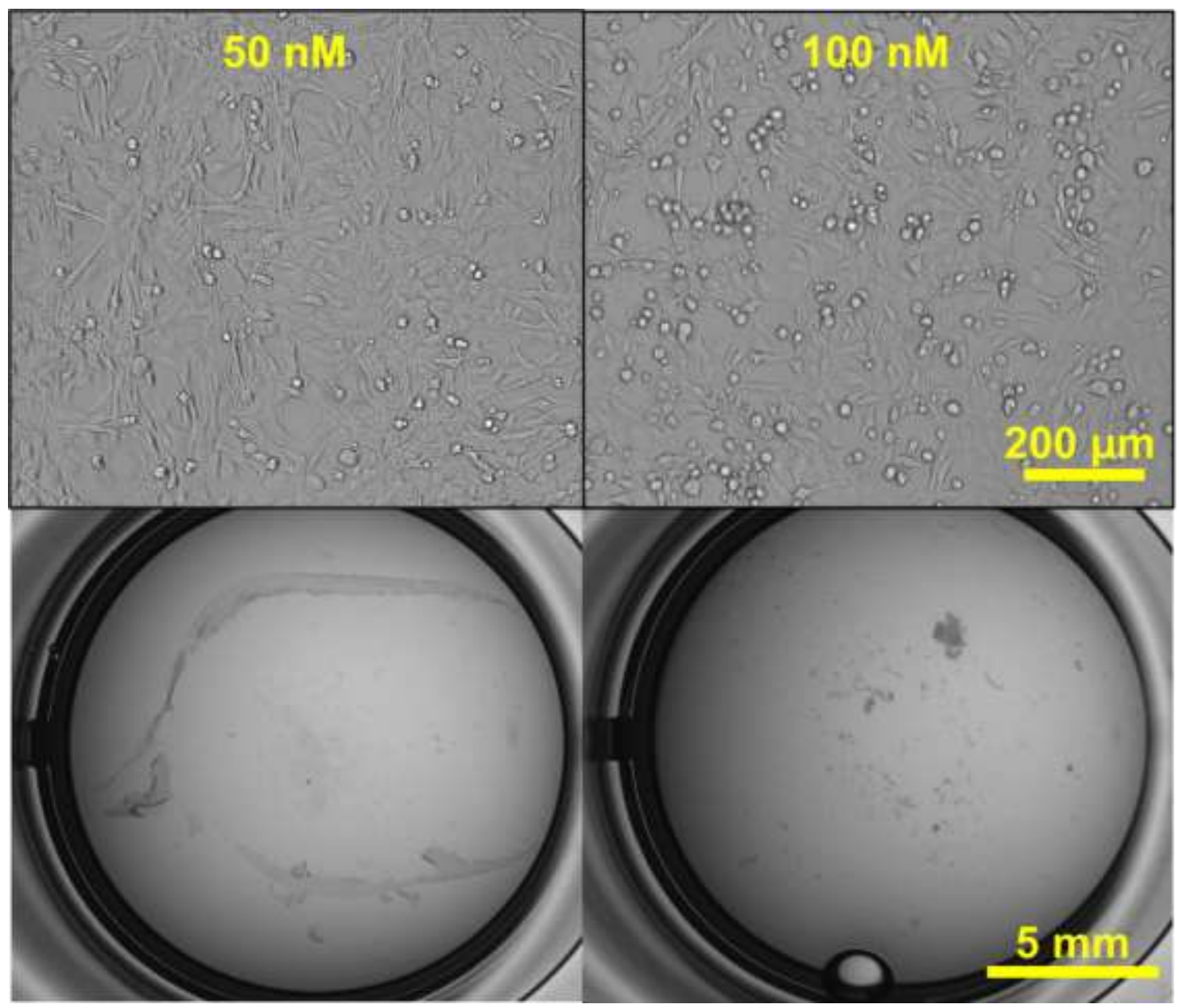

Figure S7. Delamination of layers after $24 \mathrm{hr}$ of treatment with 50 and $100 \mathrm{nM}$ solution of Latrunculin-B. $50 \mathrm{nM}$ treated groups delaminated but were fragile, $100 \mathrm{nM}$ treated ones didn't form continuous sheets. 


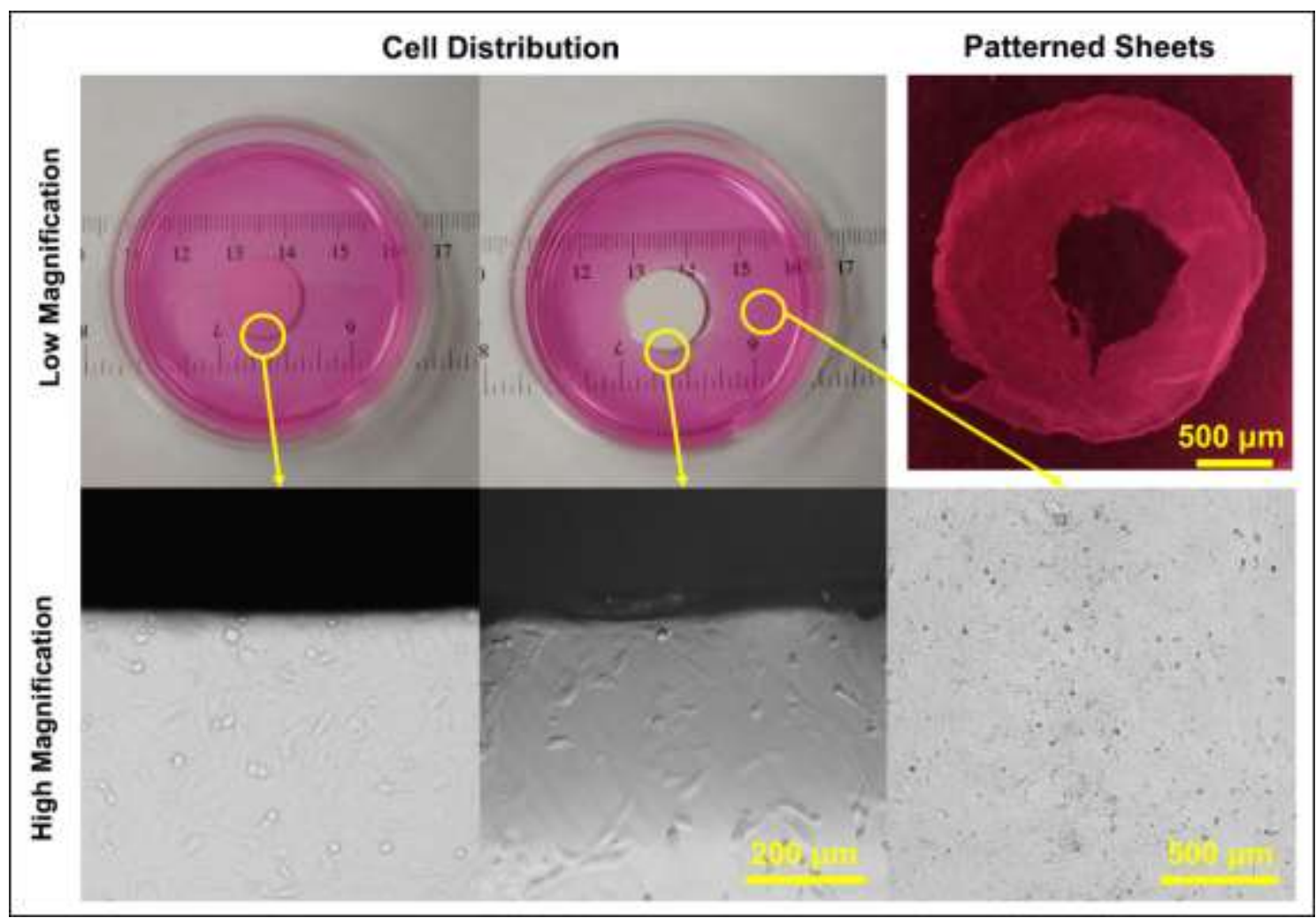

Figure S8. Forming sheets with blocked regions using Teflon tape. Teflon is highly hydrophobic and allows spaces devoid of medium and cells. If this void region is not formed, simply by shaking the dish, the surface on top of tape will be covered with media but cells won't stick to it. This will cause different coverage of cells on the non-blocked area and a better distribution is achieved when there is no void area. Sheets can only be formed in the latter case. 


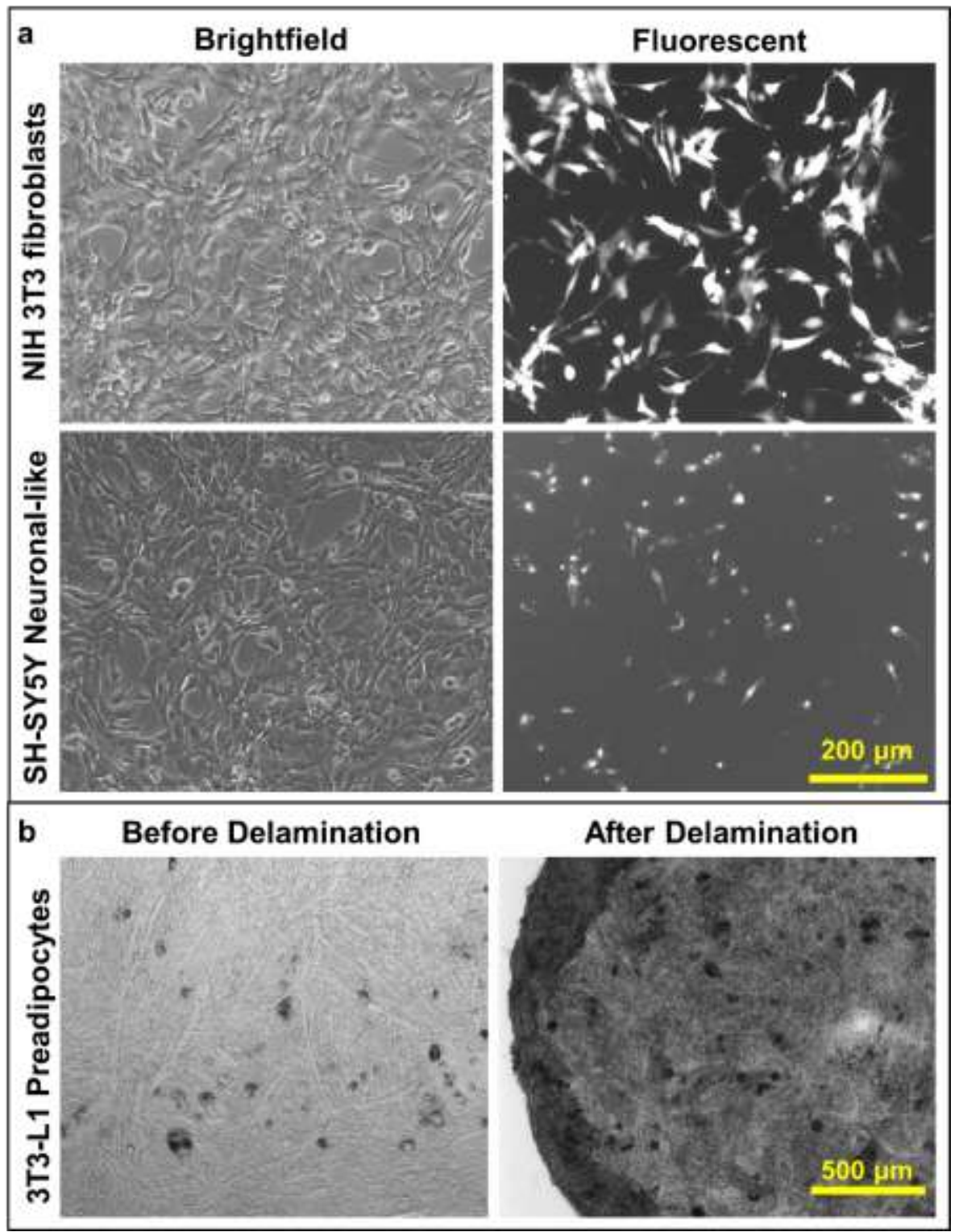

Figure S9. a) Co-culture of C2C12 cells with NIH-3T3 fibroblasts and SH-SY5Y neuronal-like cells with 5:1 ratio. b) Co-culture of $\mathrm{C} 2 \mathrm{C} 12$ myoblasts and 3T3-L1 preadipocytes before and after delamination. Starting culture with $\mathrm{C} 2 \mathrm{C} 12$ having as low as $25 \%$ of the initial population is still enough to form continuous sheets with proper integrity. 